Cite this: Phys. Chem. Chem. Phys., 2014, 16, 8332

Received 13th January 2014, Accepted 1st March 2014

DOI: $10.1039 / c 4 c p 00175 c$

www.rsc.org/pccp

\section{In-depth exploration of the photophysics of a trinuclear palladium complex}

\author{
Y. Schmitt, ${ }^{a}$ K. Chevalier, ${ }^{b}$ F. Rupp, ${ }^{b}$ M. Becherer, ${ }^{a}$ A. Grün, ${ }^{a}$ A. M. Rijs, ${ }^{c}$ F. Walz, ${ }^{d}$ \\ F. Breher, ${ }^{\star d}$ R. Diller, ${ }^{\star b}$ M. Gerhards*a and W. Klopper*e
}

\begin{abstract}
A detailed theoretical and spectroscopic study on the electronically excited states of a trinuclear palladium complex is presented both in the gas phase and solution. The application of DFT and TDDFT methods as well as a variety of spectroscopic methods to the chosen complex $\left[\mathrm{Pd}_{3}\left\{\mathrm{Si}\left(\mathrm{mt}^{\mathrm{Me}}\right)_{3}\right\}_{2}\right]\left(\mathbf{1}, \mathrm{mt}^{\mathrm{Me}}=\right.$ methimazole) leads to the first detailed analysis of the photophysics of a symmetric trinuclear complex. In combination with the calculations, energies, structures and lifetimes of the excited electronic states (with an ${ }^{3} A_{1}$ state as the lowest one) are characterized by applying the resonant-2-photon-ionization method in a molecular beam experiment as well as luminescence, time-correlated single photon counting and excited state femtosecond absorption spectroscopy in solution. These investigations are of fundamental interest to analyze photophysical properties of metal containing complexes on a molecular level.
\end{abstract}

\section{Introduction}

Electronically excited molecules are very important in many areas ranging from the single functional molecule to bulk material applications. ${ }^{1}$ Subjects meriting attention in this context include molecular photovoltaics, ${ }^{2,3}$ photocatalysis, ${ }^{4,5}$ and luminescent materials, ${ }^{6,7}$ among others. ${ }^{8-13}$ In order to properly describe and understand the molecular system of interest - in particular in view of its further development - a detailed knowledge of the involved excited states is essential. Important factors are their electronic distribution, molecular geometries, and pathways for relaxation. ${ }^{14-16}$ In order to achieve this goal, suitable spectroscopic techniques combined with quantum chemical calculations have to be applied. In view of several polynuclear transition metal complexes showing interesting properties in photochemistry and photophysics, ${ }^{14}$ we became interested in performing an in-depth analysis of one representative example which does not belong to well-elaborated molecular systems frequently used and

\footnotetext{
${ }^{a}$ Chemistry Department and Research Center Optimas, TU Kaiserslautern, Erwin-Schrödinger-Straße 52, 67663 Kaiserslautern, Germany. E-mail: gerhards@chemie.uni-kl.de; Fax: +49 631 2052750; Tel: +49 6312052537

${ }^{b}$ Physics Department, TU Kaiserslautern, Erwin-Schrödinger-Straße, 67663 Kaiserslautern, Germany.E-mail: diller@physik.uni-kl.de; Fax: +49 631 2053902; Tel: +496312052323

${ }^{c}$ Institute for Molecules and Materials/FELIX facility, Radboud University Nijmegen, Toernooiveld 7, 6525 ED Nijmegen, The Netherlands

${ }^{d}$ Institute of Inorganic Chemistry, Karlsruhe Institute of Technology (KIT), Engesser Straße 15, 76131 Karlsruhe, Germany.E-mail: breher@kit.edu; Fax: +49721 60847021; Tel: +4972160844855

${ }^{e}$ Institute of Physical Chemistry, Karlsruhe Institute of Technology (KIT), Fritz-Haber-Weg 2, 76131 Karlsruhe, Germany.E-mail: klopper@kit.edu; Fax: +49721 60847225; Tel: +4972160847263
}

investigated since several years (e.g. well-investigated MLCT systems or luminescent lanthanide complexes).

In the present article, we analyzed extensively the photophysics of a neutral trinuclear palladium complex (1, Fig. 1) ${ }^{17}$ featuring two multifunctional chelating ligands ${ }^{18}$ and an almost ideal $D_{3}$-symmetric structure. The latter aspect is important since an in-depth analysis and assignment of electronically excited states involved in the photophysics is considerably improved if the molecule (complex) of interest exhibits a certain symmetry. The chosen experimental methods cover a variety of modern spectroscopic methods ranging from steady-state spectroscopy to femtosecond absorption and fluorescence lifetime measurements, including the first application of a molecular beam (resonant ionization) method to a trimetallic complex. In order to support and verify the experimental findings, ground- and excited state

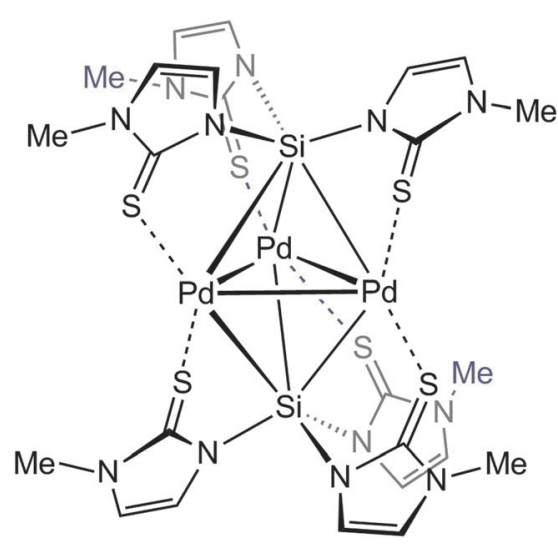

Fig. 1 The structure of $\left[\mathrm{Pd}_{3}\left\{\mathrm{Si}\left(\mathrm{mt}^{\mathrm{Me}}\right)_{3}\right\}_{2}\right](\mathbf{1})$. 
calculations were performed in the framework of density functional theory (DFT), ${ }^{19}$ also including calculations of the lifetimes of the excited states. By applying all these techniques, we were able to fully unravel the photophysics of the trimetallic complex 1. The paper is organized in the following way: the variety of chosen experimental methods is shortly presented followed by a thorough theoretical analysis. Finally the spectroscopic results are discussed by comparing them directly with the theoretical predictions.

\section{Experimental methods}

\subsection{UV/Vis/luminescence spectroscopy}

Complex 1 was synthesized according to ref. 17. Methimazole was purchased from Sigma-Aldrich with a purity grade of $99.9 \%$ and used without further purification. DMSO was used as solvent for both the complex and methimazole and purchased from Fluka for UV/Vis spectroscopy. The chosen concentrations were in the range of $10^{-5} \mathrm{~mol} \mathrm{l}^{-1}$ for both the complex and methimazole. The solutions of the complex were prepared and filled in the cuvettes (a) using the common Schlenck technique and (b) without inert gas. Argon 5.0 was used as inert gas and LUDOX ${ }^{\mathbb{R}}$ (TM-50 colloidal silica, $50 \mathrm{wt} \%$ suspension in water) was used as reference for lifetime measurements and purchased from Sigma-Aldrich.

The absorption spectra were recorded using a Perkin-Elmer Lambda 900 double beam UV/VIS/NIR spectrophotometer and cylindrical quartz cuvettes with a path length of $1 \mathrm{~cm}$. For detection of the emission a Horiba Jobin-Yvon Fluorolog 3-22 $\tau$ and $1 \mathrm{~cm} \times$ $1 \mathrm{~cm}$ quartz cuvettes were used.

\subsection{Lifetime determination}

The lifetime was determined using the time-correlated singlephoton counting technique (TCSPC). This technique offers the possibility to record the low emission intensity of $\mathbf{1}$. The set-up used for the experiments is the DeltaFlex (Horiba Jobin-Yvon) spectrometer. The sample is excited using short light pulses of a NanoLED (345 nm) and the emission monochromator was set to $675 \mathrm{~nm}$. The precision photon counting is carried out using a PPD (picosecond photon counting detection module) detection, including a fast-rise photomultiplier tube (PMT) with an integral GHz timing preamplifier, a constant fraction discriminator and a regulated high voltage supply. The instrumental response function (IRF) was collected by use of LUDOX ${ }^{\circledR}$.

\subsection{Laser desorption}

To avoid decomposition and fragmentation of complex 1, a pulsed molecular beam setup (expansion in Ar as carrier gas) equipped with a laser desorption source and a time-of-flight mass spectrometer was used. This set-up is described in detail elsewhere; ${ }^{20}$ thus only a brief introduction is given.

The complex was mixed with carbon black $(50: 50)$ and deposited on a graphite sample bar, which is placed on a translation stage, directly below the pulsed valve to provide a fresh sample at every laser shot. The sample molecules were desorbed from the graphite matrix by the fundamental of a small, pulsed Nd:YAG laser
(1064 nm, $2 \mathrm{~mJ}$ ). The neutral gas phase molecules were directly cooled in a supersonic expansion of argon.

Through a skimmer the neutral complexes entered the differentially pumped reflector-based time-of-flight mass spectrometer. There the isolated molecules interacted with the UV laser light, so the complexes were excited and finally photoionized. The ions were accelerated into the reflector time-of-flight tube and detected. By scanning the UV-laser the R2PI spectrum was obtained.

\subsection{Femtosecond time resolved UV/Vis spectroscopy}

For the transient absorption experiment the complex was dissolved in DMSO at $0.54 \mathrm{mM}$ concentration, yielding an optical density of 0.5 at the excitation wavelength $\lambda_{\mathrm{ex}}=330 \mathrm{~nm}$ and $1 \mathrm{~mm}$ path length $\left(\varepsilon_{330}=8930 \mathrm{l}(\mathrm{mol} \mathrm{cm})^{-1}\right)$. The sample was flushed with argon for 15 min before sealing the cuvette. The measurements were performed at room temperature. Before and after each experiment steady state UV/Vis spectra (JASCO V670) were recorded to exclude degradation and ensure sample integrity. The sample was rotated to exchange the excited volume between two consecutive laser-shots.

The transient absorption setup is based on a Ti:Sa chirped pulse amplifier system (Libra, Coherent, 802 nm, FWHM 90 fs, energy $3.6 \mathrm{~mJ}$ per pulse) providing UV-pump pulses by a homebuilt two-stage noncollinear parametric amplifier (NOPA). Visible continuum probe pulses were obtained by focusing a fraction of the laser output into a $5 \mathrm{~mm}$ thick calcium fluoride window, which is moved in a Lissajou figure to avoid optical damage. Pump and probe pulses were superimposed in the sample and the transmitted probe light was dispersed and detected by means of a homebuilt prism spectrograph with two 524 pixel back-thinned-CCD cameras (Stresing) for probe and reference, respectively.

Pump-induced difference spectra $\Delta \mathrm{AS}(\lambda, t)$ (= "pumped" "unpumped" absorbance) were collected as a function of probe wavelength and pump-probe delay time. The wavelength dependent time-zero of the chirped probe light and the system response time of 0.2 ps were determined using the cross-phasemodulation in a water sample.

The transient $\mathrm{UV} / \mathrm{Vis}$ data $\Delta \mathrm{AS}(\lambda, t)$ were analyzed by means of a global multiexponential fit using

$$
\Delta \mathrm{AS}(\lambda, t)=\operatorname{AS}_{0}(\lambda)+\sum_{i=1}^{N} \operatorname{AS}_{i}(\lambda) \mathrm{e}^{-t / \tau_{i}}
$$

with $\operatorname{AS}_{i}(\lambda)$ the decay associated amplitude spectra (DAS) and $\tau_{i}$ the corresponding time constants. $\operatorname{AS}_{0}(\lambda)$ represents the difference spectrum after "infinite" delay times. The fit was performed for delay times greater than $0.3 \mathrm{ps}$ to exclude coherent artefacts such as cross phase modulation during the system response time. The used fit function is a multiexponential approximation of the excited state dynamics. The DAS $\operatorname{AS}_{i}(\lambda)$ decay with the respective time constant, i.e. positive (negative) amplitude in $\operatorname{AS}_{i}(\lambda)$ represents decreasing (increasing) signal strength in the pump-induced difference spectra $\Delta \mathrm{AS}(\lambda, t)$. 


\section{Theoretical methods and calculations}

The computations reported in this article were all performed in the framework of density functional theory (DFT) using the B3LYP functional, ${ }^{21}$ the dhf-TZVP-2c basis set $^{22}$ for Pd, S, and $\mathrm{Si}$, and the def2-SV(P) basis $^{23}$ for $\mathrm{H}, \mathrm{C}$ and N. This basis is referred to as "TZVP". (Hartree-Fock theory as well as the functionals $\mathrm{BHLYP}^{24}$ and $\mathrm{BLYP}^{25,26}$ had also been tested, as well as the basis sets def2-SVP ${ }^{23}$ and def2-SVPD ${ }^{27}$ for all atoms, but the B3LYP functional reproduced the experimental absorption spectrum best while basis-set effects were negligible.) For Pd, the scalar-relativistic pseudopotential (and the corresponding spinorbit potential in two-component computations) of the energyconsistent variety was used to simulate its $[\mathrm{Ar}] 3 \mathrm{~d}^{10}$ core. $^{28}$ The computations thus comprised 436 electrons and 1022 contracted spherical-harmonic Gaussian basis functions. All computations were performed using the TURBOMOLE program package ${ }^{29}$ in $D_{3}$ symmetry. Table 1 shows selected optimized geometrical parameters of the electronic ground state in comparison with experimental X-ray data. ${ }^{17}$ The computed bond lengths are $c a .1-3 \%$ larger than found in the crystal structure.

Computations were performed both to characterize the electronic ground state and to identify the excited states originating from electronic ground-state absorption. Furthermore, the absorption spectrum resulting from a long lived excited state as well as its luminescence spectrum were calculated. Hence, the computations had to be able to provide equilibrium geometries of candidate excited states and had to be able to determine the electronic transitions (emission and absorption) from these candidate states in terms of both transition energies and oscillator strengths. A computation of the harmonic vibrational frequencies using analytic second derivatives showed that the $D_{3}$-symmetric ground-state geometry, optimized at the B3LYP/TZVP level, is a true minimum.

\subsection{Energies and ground state absorption}

From the ground-state equilibrium structure, we have computed the lowest singlet and triplet excitation energies in each of the irreducible representations $\mathrm{A}_{1}, \mathrm{~A}_{2}$, and E. Furthermore, 50 singlet $\mathrm{A}_{2}$ and 50 singlet $\mathrm{E}$ states were computed to simulate the UV absorption spectrum. These computations were performed in the framework of time-dependent density functional theory (TDDFT),${ }^{30}$ in full as well as in the Tamm-Dancoff approximation (TDA). ${ }^{31}$ The lowest singlet and triplet $\mathrm{A}_{1}$ and $\mathrm{A}_{2}$ states were also computed at the DFT level by imposing appropriate orbital occupations. We will refer to these results as obtained by the $\Delta \mathrm{SCF}$ method (difference of self-consistent field energies). ${ }^{32}$

Table 1 Selected geometrical parameters of the B3LYP/TZVP optimized equilibrium geometry of the ground state $\left({ }^{1} A_{1}\right)$ (Exptl. data from ref. 17)

\begin{tabular}{lll}
\hline Bond length & $r_{\text {B3LYP }} / \mathrm{pm}$ & $r_{\text {Expt }} / \mathrm{pm}$ \\
\hline Pd-Pd & 281.4 & $273.1(15)$ \\
Pd-Si & 243.2 & $239.8(14)$ \\
Pd-S & 244.6 & $238.6(5)$
\end{tabular}

Table 2 Vertical excitation energies (in eV) at the geometry of the ground state. The oscillator strength (length representation) is given in parentheses

\begin{tabular}{llll}
\hline State & TDDFT & TDA & $\Delta$ SCF \\
\hline $1^{1} \mathrm{~A}_{1}$ & 2.32 & 2.34 & 2.21 \\
$1^{1} \mathrm{~A}_{2}$ & $2.94(0.015)$ & $2.95(0.017)$ & 2.85 \\
$4^{1} \mathrm{~A}_{2}$ & $3.64(0.056)$ & $3.66(0.088)$ & - \\
$1^{1} \mathrm{E}$ & $2.72(0.001)$ & $2.73(0.001)$ & - \\
$4{ }^{1} \mathrm{E}$ & $3.32(0.068)$ & $3.35(0.086)$ & - \\
$5^{1} \mathrm{E}$ & $3.40(0.056)$ & $3.42(0.093)$ & - \\
$1^{3} \mathrm{~A}_{1}$ & 2.08 & 2.11 & 2.13 \\
$1^{3} \mathrm{~A}_{2}$ & 2.72 & 2.74 & 2.78 \\
$1^{3} \mathrm{E}$ & 2.54 & 2.56 & - \\
\hline
\end{tabular}

Table 2 shows the lowest vertical excitation energies as well as the most prominent transitions (of the first absorption band, see below) at the geometry of the ground state, as obtained at the TDDFT, TDA, and $\triangle \mathrm{SCF}$ levels.

Concerning the singlet excitation energies, the $\triangle \mathrm{SCF}$ results deviate slightly (ca. $0.1 \mathrm{eV}$ ) from the TDDFT and TDA energies, but apart from that the agreement between the three different approaches is remarkably good. The lowest singlet and triplet excited $\mathrm{A}_{1}$ states consist mainly (98.5\% and $97.0 \%$, respectively) of a single excitation from the doubly occupied highest occupied molecular orbital (HOMO, $37 a_{2}$ ) to the lowest unoccupied molecular orbital (LUMO, $38 a_{2}$ ). The lowest singlet and triplet excited $\mathrm{A}_{2}$ states correspond to single excitations from the first orbital below the HOMO (HOMO-1, $39 a_{1}$ ) to the LUMO (98.1\% and 97.0\%, respectively, due to a single MO pair). These (frontier) orbitals are depicted in Fig. 2. The character of the HOMO-1 is bonding between the Pd atoms, whereas the HOMO is an anti-bonding
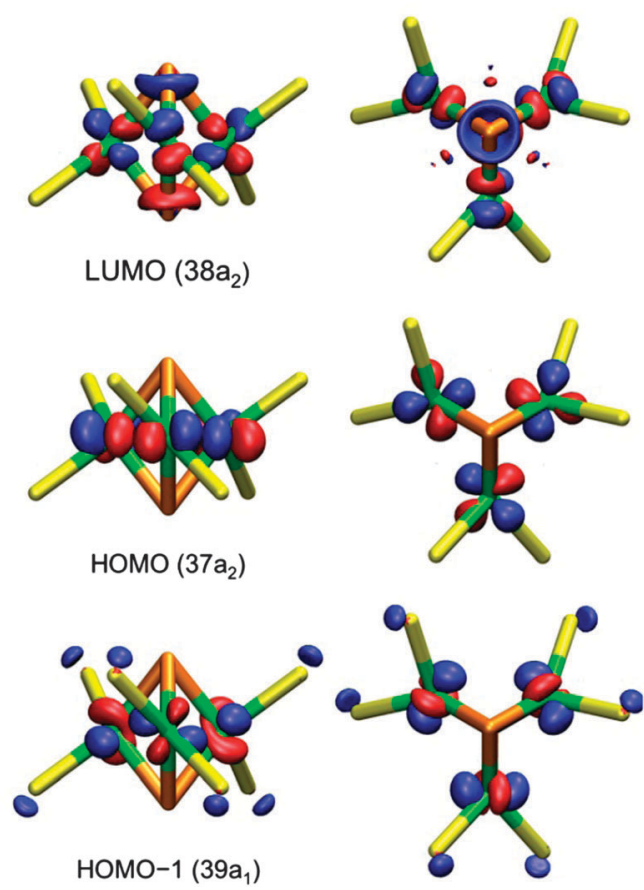

Fig. 2 Frontier orbitals as obtained at the B3LYP/TZVP level. Only the Pd (green), Si (orange) and S (yellow) atoms are shown for clarity. 


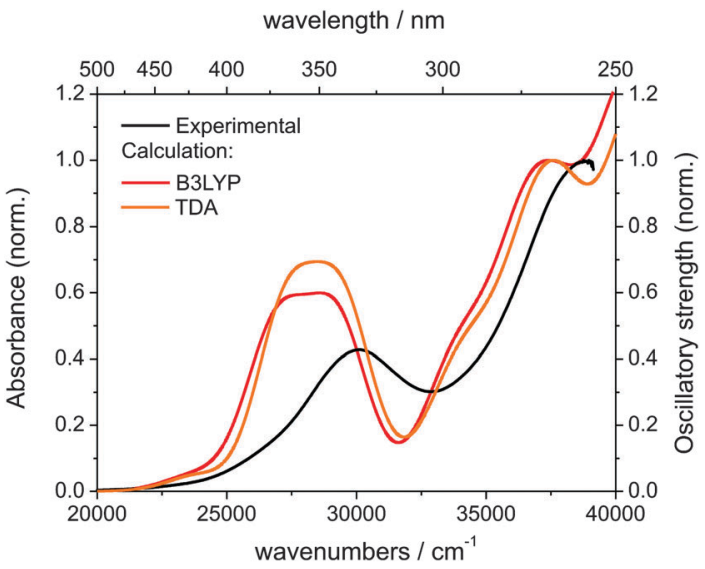

Fig. 3 Comparison of the experimental (black, DMSO solution) and calculated (red and orange) absorption spectra. For the simulation of the calculated spectra a FWHM of $2500 \mathrm{~cm}^{-1}$ was used. Calculated and experimental spectra are normalized to the strongest transition at about $266 \mathrm{~nm}$.

orbital between the Pd atoms. Furthermore, the LUMO is antibonding between Pd and Si (Fig. 2).

Fig. 3 shows the ground-state absorption spectrum obtained from computing the excitation energies and oscillator strengths of 50 singlet $\mathrm{A}_{2}$ and $50 \mathrm{E}$ states, adopting a full width at half maximum (FWHM) of $2500 \mathrm{~cm}^{-1}$. The spectrum shows a small shoulder at $c a .23700 \mathrm{~cm}^{-1}(2.94 \mathrm{eV})$, which is due to the lowest singlet $\mathrm{A}_{2}$ state (Table 2). Note that using only vertical TDDFT excitation energies is a crude approximation since vibrational effects are fully neglected. The absorption maximum at $28495 \mathrm{~cm}^{-1}(3.53 \mathrm{eV})$ (TDA) or $28585 \mathrm{~cm}^{-1}$ (3.55 eV) (TDDFT), respectively, is due to a couple of $\mathrm{A}_{2}$ and $\mathrm{E}$ states. It should be mentioned that the TDA and TDDFT approaches are in good mutual agreement.

\subsection{Excited state structure and emission spectrum}

We have optimized the geometries of the excited states at the TDDFT and $\triangle$ SCF levels, the latter however only for the states ${ }^{1} \mathrm{~A}_{1},{ }^{1} \mathrm{~A}_{2},{ }^{3} \mathrm{~A}_{1}$ and ${ }^{3} \mathrm{~A}_{2}$, with the ${ }^{3} \mathrm{~A}_{1}$ state as the lowest excited state of all. Furthermore it is important to note that the optimizations were carried out in $D_{3}$ symmetry. We have not considered possible excited-state geometries with lower symmetry. (Remark: for the ${ }^{3} \mathrm{~A}_{1}$ state, we succeeded to optimize an unsymmetric structure showing almost $C_{2}$ symmetry, whose energy was found $0.015 \mathrm{eV}$ below the $D_{3}$ structure. However, this may very well be an artifact, such as basis-set superposition effects, of the DFT calculations.) Table 3 compiles the Pd-Pd and Pd-Si bond lengths in the optimized equilibrium structures of the singlet and triplet $\mathrm{A}_{1}$ and $\mathrm{A}_{2}$ excited states. As expected, the Pd-Pd bond length in the $\mathrm{A}_{1}$ states is shorter than in the ground state ( $c f$. Table 1) while it is longer in the $\mathbf{A}_{2}$ states. In all of the optimized excited states, the Pd-Si bond length is longer than in the ground state because an electron is excited from the HOMO-1 or HOMO to the LUMO, which is anti-bonding between Pd and Si. At the geometries optimized at the TDDFT level, we have computed lifetimes of the ${ }^{1} \mathrm{~A}_{1},{ }^{1} \mathrm{~A}_{2}$,
Table 3 Optimized excited-state geometries. Bond lengths $R_{X-Y}$ are given in pm, the vertical ( $\left.\Delta E_{\text {vert }}\right)$ and adiabatic ( $\left.\Delta E_{\text {adiabat }}\right)$ emission energies are given in $\mathrm{eV}$. The oscillator strength (length representation) is given in parentheses

\begin{tabular}{llllll}
\hline State & Method & $R_{\text {Pd-Pd }}$ & $R_{\text {Pd-Si }}$ & $\Delta E_{\text {vert }}$ & $\Delta E_{\text {adiabat }}$ \\
\hline${ }^{1} \mathrm{~A}_{1}$ & TDDFT & 271.7 & 249.2 & 1.71 & 2.02 \\
& $\Delta$ SCF & 271.6 & 249.3 & 1.58 & 1.90 \\
${ }^{1} \mathrm{~A}_{2}$ & TDDFT & 287.2 & 251.7 & $2.11(0.014)$ & 2.53 \\
& $\Delta$ SCF & 287.5 & 252.1 & 1.95 & 2.40 \\
${ }^{3} \mathrm{~A}_{1}$ & TDDFT & 271.4 & 249.5 & 1.43 & 1.76 \\
& $\Delta$ SCF & 271.5 & 249.5 & 1.48 & 1.81 \\
${ }^{3} \mathrm{~A}_{2}$ & TDDFT & 287.9 & 252.3 & 1.81 & 2.28 \\
& $\Delta$ SCF & 288.2 & 252.4 & 1.84 & 2.32 \\
\hline
\end{tabular}

${ }^{3} \mathrm{~A}_{1}$, and ${ }^{3} \mathrm{~A}_{2}$ states using the BP86 functional ${ }^{33}$ in quasirelativistic two-component TDDFT calculations (hybrid functionals such as B3LYP cannot be used). ${ }^{34,35}$ For the four states $\left({ }^{1} \mathrm{~A}_{1},{ }^{1} \mathrm{~A}_{2}\right.$, ${ }^{3} \mathrm{~A}_{1}$, and ${ }^{3} \mathrm{~A}_{2}$ ), the electric dipole radiative lifetimes (in the length representation) are $5.7 \mathrm{~s}, 0.76 \mu \mathrm{s}, 0.87 \mathrm{~ms}$, and $48.5 \mathrm{~ms}$, respectively. The electric dipole radiative lifetime of the $333 \mathrm{~nm}$ absorption band can be estimated at about $80 \mathrm{~ns}$. Note that triplet lifetimes in solution can be several orders of magnitude shorter than in the gas phase. ${ }^{36}$

Furthermore, we have computed the vertical emission energy of the ${ }^{3} \mathrm{~A}_{1}$ state using spin-flip TDA (SF-TDA) ${ }^{37}$ in the local spin-density approximation. ${ }^{38}$ At the $\triangle \mathrm{SCF}$ optimized geometry, this yields an emission wavelength of $863 \mathrm{~nm}$, in good agreement with the TDDFT and $\triangle$ SCF values of 867 and $839 \mathrm{~nm}$, respectively.

Finally, we note that at the DFT level, triplet excited state energies are typically underestimated by a few tenths of an $\mathrm{eV}$. With respect to a test set of 63 triplet energies of small organic molecules, the mean error was found to be $c a .-0.4 \mathrm{eV}^{39,40}$ Assuming this error for the $863 \mathrm{~nm}$ emission, this band would shift to $675 \mathrm{~nm}$.

\section{Spectroscopic results and discussion}

In order to characterize the electronically excited states experimentally, UV/Vis spectra both in solution as well as in the gas phase have been recorded. The UV spectra of complex 1 and methimazole $\left(\mathrm{mt}^{\mathrm{Me}}\right.$, part of the ligand $\left\{\mathrm{Si}\left(\mathrm{mt}^{\mathrm{Me}}\right)_{3}\right\}^{-}$) dissolved in DMSO are shown in Fig. 4. The UV/Vis spectrum of the complex exhibits two strong transitions at 333 and $262 \mathrm{~nm}$, with the first transition at $333 \mathrm{~nm}$ showing a shoulder at $c a .400 \mathrm{~nm}$. Since the UV absorption spectrum of methimazole exhibits only one transition at $267 \mathrm{~nm}$, it can be assumed that the transition of the complex at $262 \mathrm{~nm}$ has significant contributions from ligand excitations, whereas the bathochromic transitions are characteristic for the $\mathrm{Pd}_{3}$ core structure.

The shape of the experimentally observed absorption spectrum of the complex is in very good agreement with the calculated spectrum, only the maxima are shifted by about 1200 to $1800 \mathrm{~cm}^{-1}$ (cf. Fig. 3).

In order to understand the influence of the solvent on the absorption, a one-color resonant two-photon ionization (R2PI) spectrum has been recorded in the region at around 


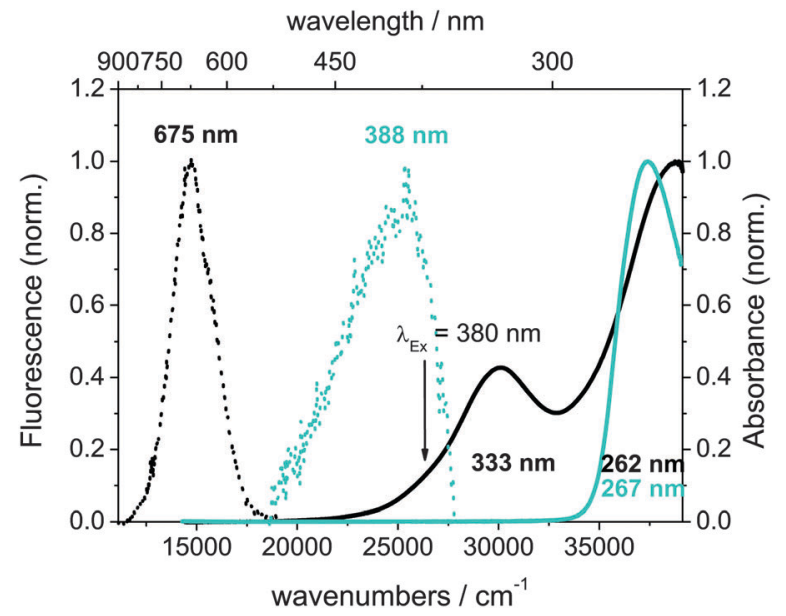

Fig. 4 Normalized absorption (solid line) and emission (dashed line) spectra of 1 (black) and methimazole (cyan) dissolved in DMSO. The arrow marks the excitation wavelength for the depicted emission spectrum of the complex. The emission spectrum of 1 obtained by excitation at $333 \mathrm{~nm}$ is identical. In the case of the emission spectrum of methimazole, an excitation wavelength of $275 \mathrm{~nm}$ has been chosen.

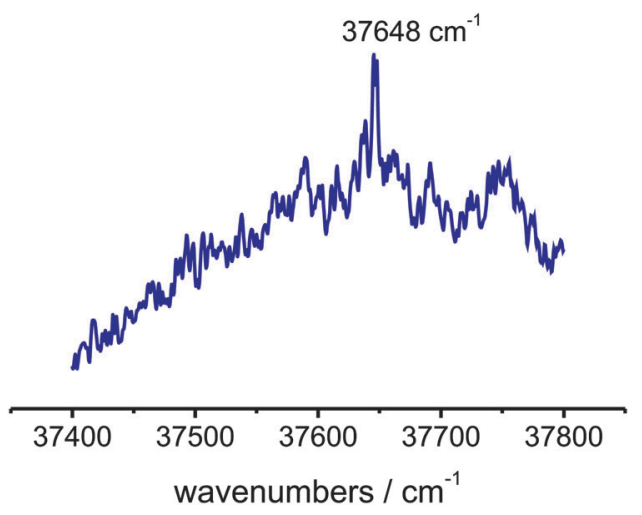

Fig. 5 One color R2PI spectrum of 1 in a molecular beam experiment from 37400 to $37800 \mathrm{~cm}^{-1}$, with a maximum at $37648 \mathrm{~cm}^{-1}(266 \mathrm{~nm})$.

$266 \mathrm{~nm}$ ( $c f$. Fig. 5), i.e. the first photon leads to an absorption to the electronically excited state, whereas the second photon ionizes the molecule. (In the region of $266 \mathrm{~nm}$ a one color $(1+1)$-R2PI spectrum can be recorded.) Due to the low vapor pressure and the thermal instabilities of complex 1, a laser desorption source has been used to bring the complex into the gas phase ( $c f$. Experimental methods). Fig. 5 shows the absorption spectrum of the Pd-complex under isolated conditions. The observed maximum of the spectrum at $266 \mathrm{~nm}$ is in excellent agreement with the calculated one.

Therefore, it can be concluded that the shift between the calculated spectrum (calculated for isolated molecules in the gas phase) and the UV spectrum in solution arises from a solvent effect. Thus, the gas phase spectrum further supports the quality of the DFT calculations.

In an additional experiment, luminescence spectra have been recorded for both the complex and methimazole. The corresponding luminescence obtained for the complex $\mathbf{1}$ by using an excitation wavelength of $380 \mathrm{~nm}$ is shown in Fig. 4 . A weak and very symmetric emission transition at $675 \mathrm{~nm}$ is observed indicating a large bathochromic shift which is also postulated from the calculations (note that the luminescence of methimazole at $388 \mathrm{~nm}$ is significantly different from the one of complex 1, $c f$. Fig. 4). In order to get a further characterization of the excited state of the complex, the lifetime of the transition at $675 \mathrm{~nm}$ was determined. Due to the weakness of the transition, the time correlated single photon counting method (TCSPC, $c f$. Experimental methods) has been chosen leading to a lifetime of $\tau$ of $222 \pm 3 \mathrm{~ns}$ if the solution is not purged with argon. If oxygen is removed by purging the solution with argon, the lifetime increases significantly to about 1.4-1.7 $\mu \mathrm{s}$. These experimental results do not only indicate strong oxygen quenching, but also they are not typical for a singlet state, i.e. the long lifetime indicates the formation of a triplet state. This is again in agreement with the calculations postulating a triplet sate $\left({ }^{3} \mathrm{~A}_{1}\right)$ as the lowest level in the excitation manifold. However, by looking at the absolute values of the calculated transition energies, the ${ }^{3} \mathrm{~A}_{2}$ state fits best to the experimentally observed value. On the other hand, by taking into account the possible errors of the triplet-state calculations mentioned above, the "corrected" calculated value of $675 \mathrm{~nm}$ for the ${ }^{3} \mathrm{~A}_{1}$ state would also perfectly fit the experimental energy. In order to find out if indeed the ${ }^{3} \mathrm{~A}_{1}$ and not the ${ }^{3} \mathrm{~A}_{2}$ state is responsible for the experimentally observed emission, the excited states were further characterized by applying femtosecond time resolved UV/Vis excited state absorption spectroscopy.

After photoexcitation at $330 \mathrm{~nm}$ the observed absorption changes are dominated by an instantaneous bleach in the region of the electronic ground state (GS) absorption $(\lambda<370 \mathrm{~nm})$ and a concomitant build up of excited state absorption (ESA) at 370-750 $\mathrm{nm}$. Both bleach and ESA appear within 1 ps and persist without a measurable change for 700 ps (longest experimental delay time). These kinetics are summarized in Fig. 6 by means of the decay associated amplitude spectra $\mathrm{AS}_{0}$ and $\mathrm{AS}_{1}$ as a result of the global kinetic analysis. For comparison, the stationary electronic ground state absorption spectrum is shown.

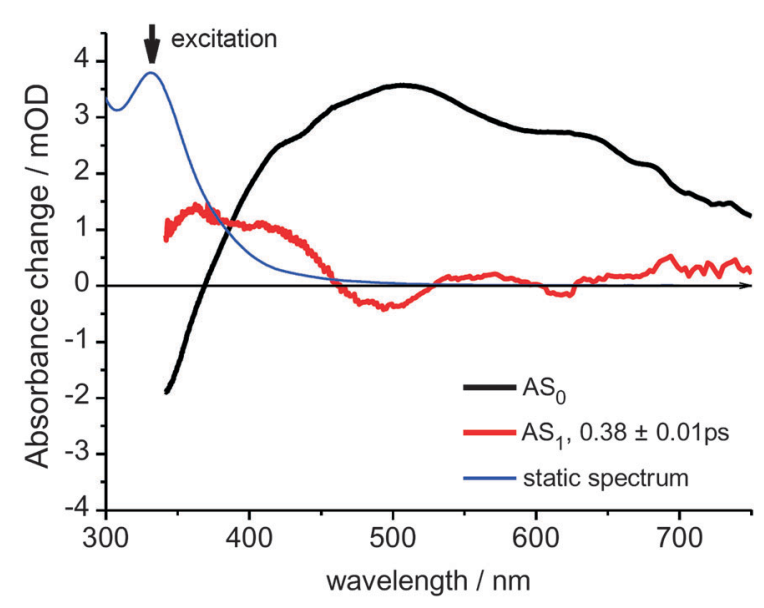

Fig. 6 Decay associated spectra $\mathrm{AS}_{0}$ and $\mathrm{AS}_{1}$ of 1 in DMSO; excitation at $330 \mathrm{~nm}$. Blue: static electronic ground state absorption (arbitrary units). 


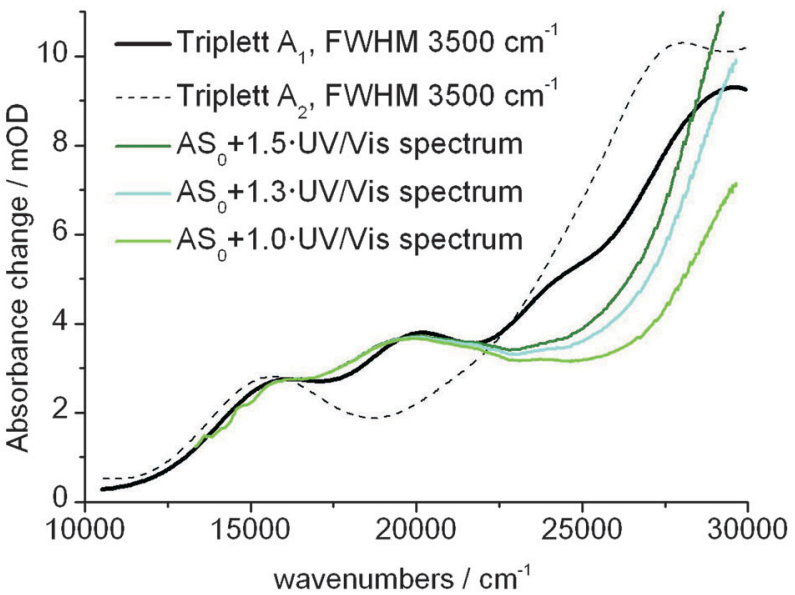

Fig. 7 Comparison of calculated and experimental excited electronic state absorption spectra. Black solid (dashed): simulation of the ${ }^{3} A_{1}\left({ }^{3} A_{2}\right)$ absorption spectrum based on ca. 150 transitions to upper electronic states (transition energy, transition strength and Gaussian widths of $3500 \mathrm{~cm}^{-1}$ ). The overall amplitudes of the calculated spectra are scaled to match the experimental spectrum (intensity), especially in the region far off ground state absorption $\left(\lambda>400 \mathrm{~nm}, \tilde{v}<25000 \mathrm{~cm}^{-1}\right)$.

The absorption difference between the long lived excited state and the depleted ground state population is represented by $\mathrm{AS}_{0}$, i.e. positive excited state absorption (ESA) is observed above ca. $370 \mathrm{~nm}$ and negative ground state absorption below $370 \mathrm{~nm}$. The excited state absorption spectrum in this presentation clearly shows peaks/shoulders at 420 (23 800), 504 (19800), $630(16000)$ and also $680(14700) \mathrm{nm}\left(\mathrm{cm}^{-1}\right)$ (see also Fig. 7). The small amplitude $\mathrm{AS}_{1}$ decays with 0.38 ps and exhibits positive (transient ESA) contributions at around $340-460 \mathrm{~nm}$ and noisy features above $460 \mathrm{~nm}$. This indicates very fast processes on the excited state potential energy surface(s) on this time scale. Thus, the femtosecond experiment shows that within less than $1 \mathrm{ps}$ after excitation at $330 \mathrm{~nm}$ a very long lived ( $\gg 0.7 \mathrm{~ns}$ ) excited electronic state is formed. No further dynamics are observed on this time scale.

In order to compare these experimental results with theoretical predictions, we have computed the absorption spectra with respect to electronic excitations out of the corresponding singlet and triplet excited $\mathrm{A}_{1}$ and $\mathrm{A}_{2}$ states. Such computations are possible using the TURBOMOLE program package at the TDA level.

Fig. 7 shows in black the simulation of the ${ }^{3} \mathrm{~A}_{1}$ absorption spectrum based on about 150 ( $c a .50$ significantly contributing) transitions to upper electronic states (transition energy, transition strength and Gaussian FWHM of $3500 \mathrm{~cm}^{-1}$ ). This spectrum is compared with the experimental excited state absorption spectra derived from the decay associated amplitude spectrum $\mathrm{AS}_{0}$ by adding slightly varying (because not exactly known) contributions of the electronic ground state absorption spectrum. The overall amplitude of the calculated spectrum is scaled to match the experimental spectrum (intensity) in the region far off the ground state absorption $\left(\lambda>400 \mathrm{~nm}, \tilde{v}<25000 \mathrm{~cm}^{-1}\right)$. Obviously the agreement between experiment and theory is very good: both the experimental transition energies and the intensities are very well reproduced by the simulation. The only

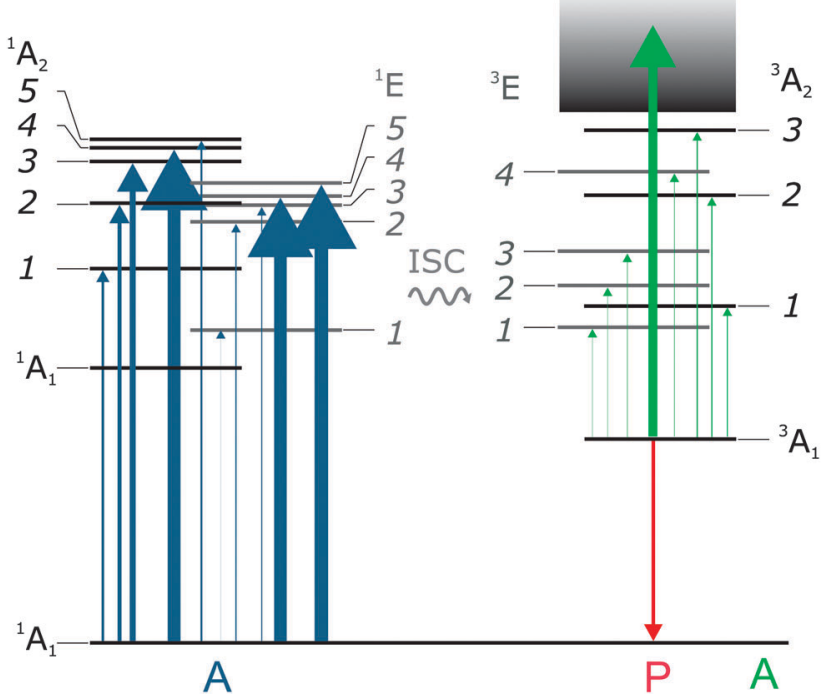

Fig. 8 Schematic diagram of the electronic states involved in the photophysics of complex 1 (based on the TDA calculations). The oscillatory strengths of the different transitions are indicated by the width of the corresponding arrows. The transitions which are responsible for the first absorption band in Fig. 3 are marked in blue ( $A$ in blue), the green colored arrows indicate the excited triplet state absorptions and the red arrows show the luminescence (phosphorescence) of the complex from the longlived triplet ground state ${ }^{3} \mathrm{~A}_{1}$.

significant deviation is that of the (relative) intensity mismatch (30\% effect) at around $24000 \mathrm{~cm}^{-1}$. However, the analogous comparison with the calculated ${ }^{3} \mathrm{~A}_{2}$ state yields much less similarity, in particular in the region from about 17500 to $22500 \mathrm{~cm}^{-1}$; the calculated spectrum for the ${ }^{3} \mathrm{~A}_{2}$ state and the experimental spectrum go in opposite directions. Thus, the transient absorption experiment clearly favors the ${ }^{3} \mathrm{~A}_{1}$ state (and not the ${ }^{3} \mathrm{~A}_{2}$ state) as the state populated for nanoseconds after photoexcitation at $330 \mathrm{~nm}$, i.e. the luminescence should also start from the ${ }^{3} \mathrm{~A}_{1}$ and not from the ${ }^{3} \mathrm{~A}_{2}$ state. The strong deviation concerning state energy between the "uncorrected" calculations and the luminescence experiment cannot only be explained by solvent induced shifts (as in the case of the absorption spectrum). That the emission process is the only one which undergoes a change in multiplicity has also to be taken into account, i.e. calculations can describe absorption within one multiplicity very well but a change in multiplicity leads to stronger deviations explaining the difference between observed and calculated emission maxima. ${ }^{22,23}$ Thus, only the chosen set of multiple spectroscopic and theoretical methods leads to a thorough analysis of the electronically excited states of the neutral Pd complex 1.

\section{Conclusion}

In this article we present the first detailed theoretical and spectroscopic investigations on the electronically excited states of a symmetric trinuclear palladium complex. An extensive theoretical characterization at the DFT and TDDFT levels as 
well as a large variety of experimental techniques ranging from steady-state absorption, luminescence, excited state absorption, lifetime measurements up to molecular beam investigations offer a unique insight into the detailed photophysics of the investigated complex $\left[\mathrm{Pd}_{3}\left\{\mathrm{Si}\left(\mathrm{mt}^{\mathrm{Me}}\right)_{3}\right\}_{2}\right]$ (1). Due to its $D_{3}$ symmetry, the complex was found to be an ideal example to analyze detailed aspects of its photophysics. The extensive spectroscopic and theoretical investigations can be summarized in the diagram of Fig. 8. In particular, these analyses especially lead to a detailed characterization of the lowest lying triplet state ${ }^{3} \mathrm{~A}_{1}$ with respect to its energy, lifetime, and structure. Investigations on intermediate or long-lived excited states of this type are of high interest for general fundamental research questions with respect to the photophysics and electronic properties of metal complexes which are discussed in modern applications like photovoltaic cells or photochemical devices.

\section{Acknowledgements}

Financial support from the Collaborative Research Center CRC/ Transregio 88, "Cooperative effects in homo- and heterometallic complexes (3MET)” is gratefully acknowledged (Projects B4, C1, $\mathrm{C} 2, \mathrm{C} 4)$.

\section{References}

1 Electron Transfer in Chemistry, ed. V. Balzani, Wiley-VCH, Weinheim, 2001.

2 M. Grätzel, Acc. Chem. Res., 2009, 42, 1788.

3 A. Hagfeldt and M. Grätzel, Acc. Chem. Res., 2000, 33, 269.

4 A. J. Esswein and D. G. Nocera, Chem. Rev., 2007, 107, 4022.

5 M. Fagnoni, D. Dondi, D. Ravelli and A. Albini, Chem. Rev., 2007, 107, 2725.

6 J.-C. G. Bünzli and C. Piguet, Chem. Soc. Rev., 2005, 34, 1048.

7 S. V. Eliseeva and J.-C. G. Bünzli, Chem. Soc. Rev., 2010, 39, 189.

8 M. A. Fox, Acc. Chem. Res., 2012, 45, 1875.

9 J. O. Johansson and E. E. B. Campbell, Chem. Soc. Rev., 2013, 42, 5661.

10 Z. Liu, W. He and Z. Guo, Chem. Soc. Rev., 2013, 42, 1568.

11 P. S. Wagenknecht and P. C. Ford, Coord. Chem. Rev., 2011, 255, 591.

12 O. S. Wenger, Acc. Chem. Res., 2013, 46, 1517.

13 J. Zhao, W. Wu, J. Sun and S. Guo, Chem. Soc. Rev., 2013, 42, 5323.

14 Metal Clusters in Chemistry, ed. P. Braunstein, L. A. Oro and R. R. Raithby, Wiley-VCH, Weinheim, 1999, vol. 1-3.
15 J. F. Endicott, in Inorganic Electronic Structure and Spectroscopy, ed. E. I. Solomon and A. B. P. Lever, Wiley, 2006, vol. II, pp. 291-341.

16 J. K. McCusker, Acc. Chem. Res., 2003, 36, 876.

17 F. Armbruster, J. Meyer, A. Baldes, P. Ona-Burgos, I. Fernández and F. Breher, Chem. Commun., 2011, 47, 221.

18 I. Kuzu, I. Krummenacher, J. Meyer, F. Armbruster and F. Breher, Dalton Trans., 2008, 5836.

19 C. Adamo and D. Jacquemin, Chem. Soc. Rev., 2013, 42, 845. 20 A. M. Rijs, E. R. Kay, D. A. Leigh and W. J. Buma, J. Phys. Chem. A, 2011, 115, 9669.

21 A. D. Becke, J. Chem. Phys., 1993, 98, 5648.

22 F. Weigend and A. Baldes, J. Chem. Phys., 2010, 133, 174102.

23 F. Weigend and R. Ahlrichs, Phys. Chem. Chem. Phys., 2005, 7, 3297.

24 A. D. Becke, J. Chem. Phys., 1993, 98, 1372.

25 A. D. Becke, Phys. Rev. A: At., Mol., Opt. Phys., 1988, 38, 3098.

26 C. Lee, W. Yang and R. G. Parr, Phys. Rev. B: Condens. Matter Mater. Phys., 1988, 37, 785.

27 D. Rappoport and F. Furche, J. Chem. Phys., 2010, 133, 134105.

28 K. A. Peterson, D. Figgen, M. Dolg and H. Stoll, J. Chem. Phys., 2007, 126, 124101.

29 TURBOMOLE V6.4, a development of Universität Karlsruhe (TH) and Forschungszentrum Karlsruhe GmbH, 1989-2007, TURBOMOLE GmbH, since 2007. Available from http:// www.turbomole.com, 2012.

30 F. Furche and D. Rappoport, in Computational Photochemistry, ed. M. Olivucci, Elsevier, 2005, vol. 16, p. 93.

31 S. Hirata and M. Head-Gordon, Chem. Phys. Lett., 1999, 314, 291.

32 T. Kowalczyk, S. R. Yost and T. Van Voorhis, J. Chem. Phys., 2011, 134, 054128.

33 J. P. Perdew, Phys. Rev. B: Condens. Matter Mater. Phys., 1986, 33, 8822 .

34 M. Kühn and F. Weigend, J. Chem. Theory Comput., 2013, 9, 5341.

35 M. Kühn and F. Weigend, to be published.

36 J.-F. Greisch, M. E. Harding, M. Kordel, W. Klopper, M. M. Kappes and D. Schooss, Phys. Chem. Chem. Phys., 2013, 15, 8162.

37 M. Kühn and F. Weigend, ChemPhysChem, 2011, 12, 333128. 38 J. P. Perdew and Y. Wang, Phys. Rev. B: Condens. Matter Mater. Phys., 1992, 45, 13244.

39 D. Jacquemin, E. A. Perpète, I. Ciofini and C. J. Adamo, J. Chem. Theory Comput., 2010, 6, 1532.

40 M. J. G. Peach, M. J. Williamson and D. J. Tozer, J. Chem. Theory Comput., 2011, 7, 3578. 